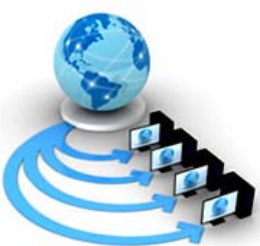

Volume 8, No. 7, July - August 2017

Available Online at www.ijarcs.info

\title{
QOS PROVISIONING FUZZY BASED STABLE ELECTION PROTOCOL IN WSN
}

\author{
Amarpreet Kaur \\ M.tech Electronics and Communication Engineering \\ Rayat Institute of Engineering and Information Technology \\ Railmajra, India
}

\author{
Manwinder Singh \\ Associate Prof in Electronics and Communication \\ Engineering \\ Rayat Institute of engineering and Information Technology \\ Railmajra, India
}

\begin{abstract}
In a Wireless Sensor Network, the most significant parameter of sensor nodes is Energy. One of the demanding concerns in WSNs is Energy Efficiency. The solution to this problem is data aggregation which will decrease the network traffic. Data aggregation can be achieved by using Clustering Schemes which is an essential research area, because it provides multiple benefits such as it upgrades constancy and reduces the network overhead that prolongs the lifespan of the network. The major idea behind the clustering scheme is to deploy the sensor nodes in the network, compress the data gathered from the sensor nodes and then transfers the gathered data to the sink. Cluster head selection is the fundamental part in this procedure. Several Cluster Head Selection Techniques are considered in this paper. This paper proposed a fuzzy interface system which has used to choose a cluster head on the basis of different input parameters given to it. In this method, automated method has followed for the selection. Experimental analysis have performed between proposed, SEP and LEACH protocol on the basis of number of alive nodes, number of dead nodes and throughput. From the analysis, it has been concluded that the proposed technique enhances the lifetime of the network in comparison with other traditional methods.
\end{abstract}

Keywords: Energy Efficient protocol, Clustering, Clusterhead selection, Fuzzy interface system, LEACH, SEP, Multi-hoping

\section{INTRODUCTION}

Effective usage of the restricted resources of sensor nodes is the major purpose of WSNs routing protocols in order to increase the network's lifespan [1]. On the basis of the requirements, several routing strategies can be adopted for various applications. Routing techniques can be improved and adapted for particular application. The energy efficient routing protocols in WSNs can usually be classified based upon the structure of the network such as: Data Centric, Hierarchical and Location based routing [2].

i. In data-centric approach, each node is comparable in function and correlate in a query routing acknowledged from the base station to the event.

ii. In hierarchical method, several nodes have additional responsibilities in the network so as to decrease the load on other nodes

iii. In location based routing, the information related to the locations of sensor nodes is exploited for routing the query from the base station to the event [3].

Clustering enhances the network life of a sensor by diminishing the consumption of energy. A sensor network is scalable with the help of forming clusters. A cluster leader is known as Cluster Head (CH) [4]. The cluster sensor nominated a $\mathrm{CH}$ or it is pre-assigned by a network designer. In order to maintain the scalability and effective communication, several algorithms for clustering were purposely designed for WSNs. The cluster based routing model executes energyefficient routing in WSNs [5]. In case of hierarchical design, nodes with higher energy (CHs) process and transfer information while the nodes having low energy perform sensing. PEGASIS, Low Energy Adaptive Cluster Hierarchy protocol (LEACH), TEEN and APTEEN are some of the examples of clustering algorithms [6][7].

i. With the help of clustering, size of the routing table decreases stored at specific nodes by localizing a route set up in a cluster. ii. The lifespan of sensors battery can be extended by $\mathrm{CH}$. It also increases the network's life by performing improved management techniques.

iii. The bandwidth of communication is preserved by clustering because it confines range of inter-cluster communications to $\mathrm{CHs}$ by evading redundant message exchange among sensor nodes.

iv. Clustering cuts on maintenance overhead of topology. Sensors concern to connect with their CHs

v. By setting up activities in a cluster, a $\mathrm{CH}$ decreases the consumption rate of energy.

vi. A $\mathrm{CH}$ processes data aggregation in its cluster and reduces unnecessary packets.

\section{- Clustering strategies}

A lot of clustering schemes for enhancing the lifetime of network are described signifying unusual techniques for the selection of cluster head and its responsibility among the sensor nodes, utilizing several parameters that are given below [8]:

1) Number of Clusters: According to the algorithms for cluster head selection, number of clusters may be different. This count will be the predestined one in few cases.

2) Intra-cluster: By this parameter, it means that the communication which occurs between the node and Cluster head may be achieved through a single-hop or multi-hop communication.

3) Nodes and CH Mobility: in the case where sensor nodes are having the feature of mobility, cluster formation can be changed.

4) Node Type and Roles: the types of nodes matters in case of cluster head selection. There are two types of nature of nodes such as homogeneous and heterogeneous [9]. Each sensor node has equal abilities such as energy and structures. Whereas in heterogeneous the configuration of nodes are unique from one another. 
5) Cluster Head Selection: Among the deployed nodes, cluster heads are selected based upon certain principles such as residual energy, connectivity, mobility and cost of communication.

6) Multiple Levels: this type of clustering method has done in order to improve the distribution of energy in huge networks [10].

7) Overlapping: Many of the protocols do not support for overlapping of dissimilar clusters.

On the basis of these parameters, the cluster head selection techniques may generally be classified into deterministic, adaptive and hybrid metric [11][12][13][14]. The particular aspects of the sensor nodes such as Node ID i.e. identification number, degree of node i.e. their count of neighbors are considered in deterministic strategies whereas in adaptive designs, the residual energy, initial energy and the energy degenerated during final round are utilized to choose their job during several data assembly rounds. The adaptive techniques can be further categorized as base station or self -organized based upon the initialization process of cluster head selection [8]. On the other hand, parameters which are considered to decide the function of a sensor node are used to classify the Probabilistic strategies. Lastly, the combination of deterministic and adaptive strategies is also used by some other schemes considered as hybrid techniques.

\section{- Multi hop communication}

The wireless sensor network makes use of either single hop communication model or multi hop communication model [15].

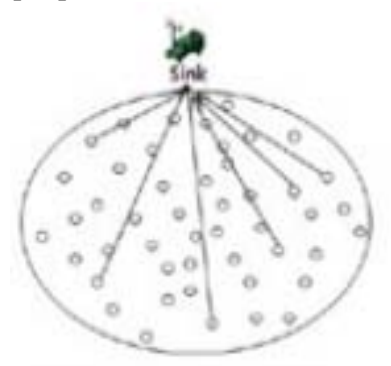

(a) Single-hop flat model

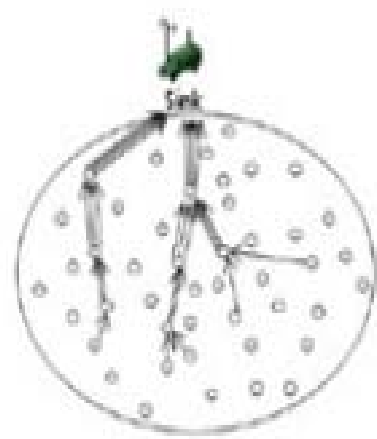

(c)Multi-hop Flat model

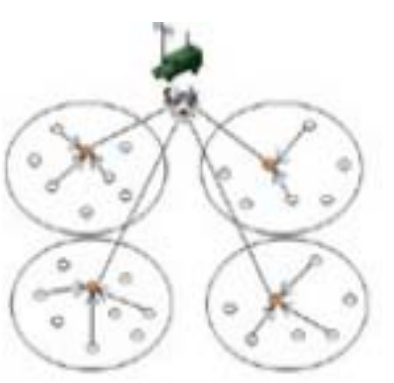

(b) Single-hop Clustering Model

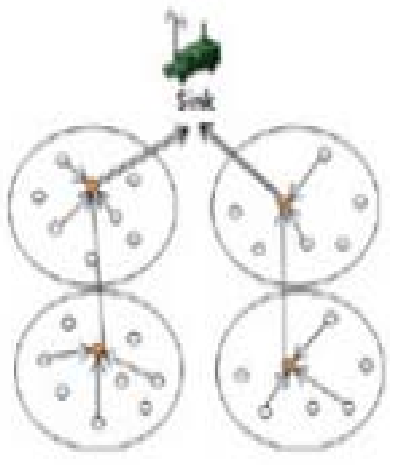

( d) Mutli-hop clustering model
Figure 1 Classification of Clustering Topologies in WSN

In both of the model, the amount of energy consumption plays a crucial part where utilization of energy is directly relative to the distance of sensor nodes in the network [16] [17]. The single hop model is costly in comparison with multi hop model in terms of energy consumption [18]. Alternatively, most of the routing algorithms used multi hop model as it utilizes energy proficiently.

\section{PROBLEM FORMULATION}

Many protocols have been proposed till now to reduce the energy as it is the vital part of wireless communication. In the existing techniques, cluster head has selected on the random basis without considering distance and energy consumption which results into an ineffective communication of nodes. Follow-on this technique, a fuzzy based system has proposed which are based upon the three parameters such as:

- $\quad$ Battery Energy

- Distance of node with the sink

- Density

The number of parameters is lesser so the selection of clusterhead has not done properly. Moreover, the node which is nearer to the sink has selected as clusterhead which causes the long distance with other nodes. Secondly, clusterhead of other clusters is also away from the sink which consumes high energy and degrades the system.

\section{PROPOSED WORK}

In order to pass up the problems of the proposed work, the existing fuzzy system is upgraded, in which number of parameters are enhanced such as:

- $\quad$ Battery Energy

- Distance of node with the sink

- Density

- Overall mean Distance

- Multi-hoping

Furthermore, selection of clusterhead has done through computing overall mean value of the other nodes. Thus, selected node's distance is calculated with other nodes to choose appropriate clusterhead in the related clusters.

Second problem mentioned in the problem formulation is resolved through proposing a concept of multi-hoping in which clusterhead of the cluster choose the nearest node in the other clusters to send their data to the sink.

\section{METHODOLOGY}

The figure below shows the block diagram of the proposed work.

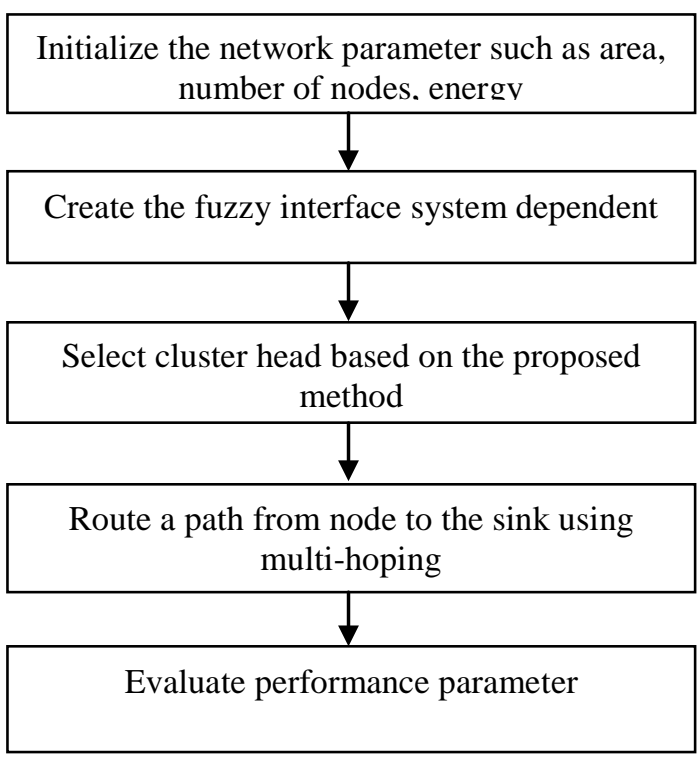

Figure 2 Block diagram of proposed work.

The proposed work selects the clusterhead on the basis of five different parameters. By mean of introducing more than one 
parameter, clusterhead can be chosen effectively. The methodology follows by the proposed work is as follows:

1. First step is to initialize the parameters for network configuration. The parameters are as follows:

a) Area of the network

b) Number of nodes in the network

c) Location for sensor node deployment

d) Energy

2. In this step, create Fuzzy Interface system in order to enhance the number of parameters.

3. Next step is to perform clustering and cluster head selection. The cluster heads will be selected on the basis of following basis;

a) Overall mean Distance.

b) Distance of node with other nodes.

4. Route a path from a node to the sink using the concept of multi-hoping where cluster head choose the nearest node to send its data to the sink.

5. After transferring of data, evaluate the performance parameter.

\section{RESULTS AND DISCUSSION}

This section represents the results that are obtained after implementing the proposed work which is done in MATLAB. There are some graphs in this section which proves the efficiency of proposed technique with respect to various aspects such as number of dead nodes, number of alive nodes and throughput. In the figure 3, different techniques have been compared with each other in terms of parameter delay. It has been shown that the delay in the proposed technique is less in comparison with other techniques such as SEP and LEACH.

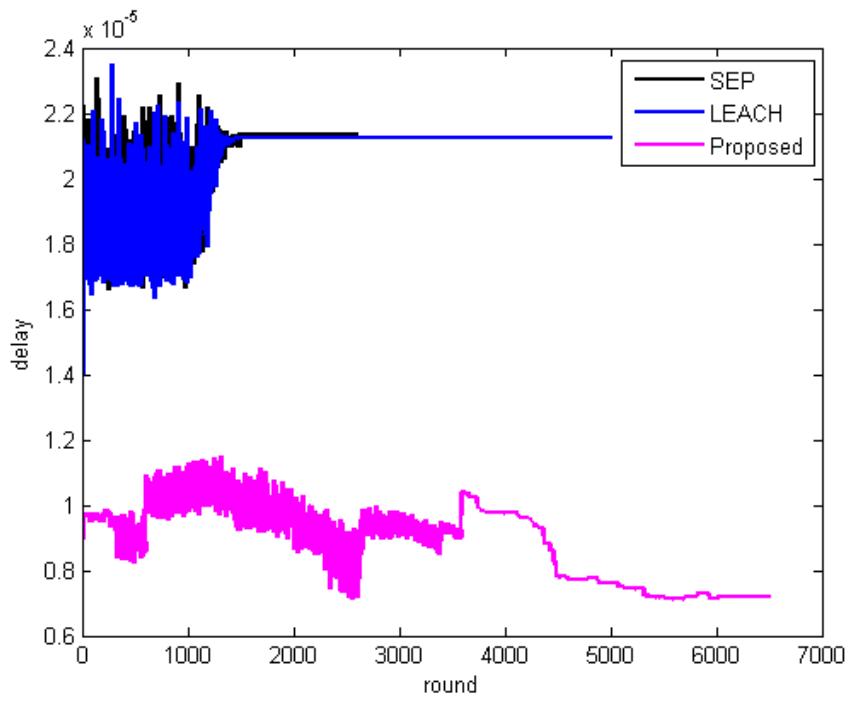

Figure 3 comparisons of different techniques in terms of delay

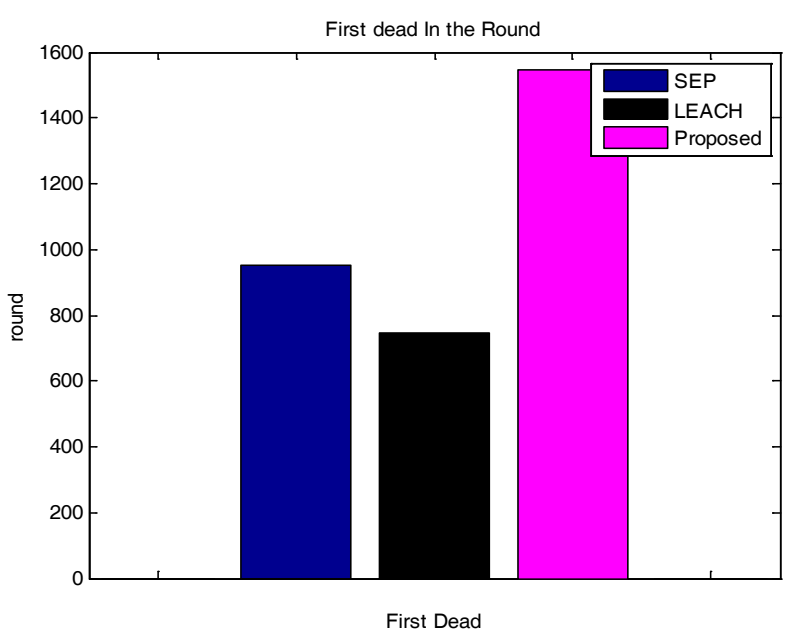

Figure 4 First Dead node in different techniques

The above figure shows the first dead node in the network using different techniques such as SEP, LEACH and proposed technique. In the SEP, first node dead after 900 rounds and in $\mathrm{LEACH}$, it has been dead at 700 rounds which shows the lifetime of the network i.e. low. In comparison with these techniques, proposed technique outperforms where the very first node is dead after 1400 rounds which is quite better than other techniques. Moreover, it shows that the lifetime of the network is superior.

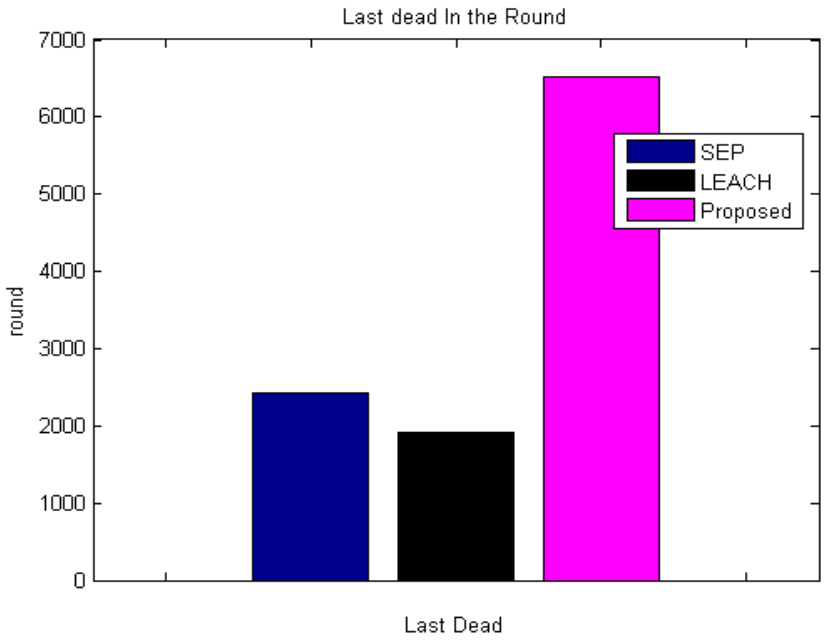

Figure 5 Last dead node in the network

The figure 5 shows the Last dead node in the network using different techniques such as SEP, LEACH and proposed technique. In the SEP, all the nodes have been dead at 2500 rounds and in LEACH, it has been dead at 1900 rounds which show the lifetime of the network i.e. low. In comparison with these techniques, proposed technique outperforms where the nodes perform transmission till 6500 rounds which is reasonably improved than other techniques. 


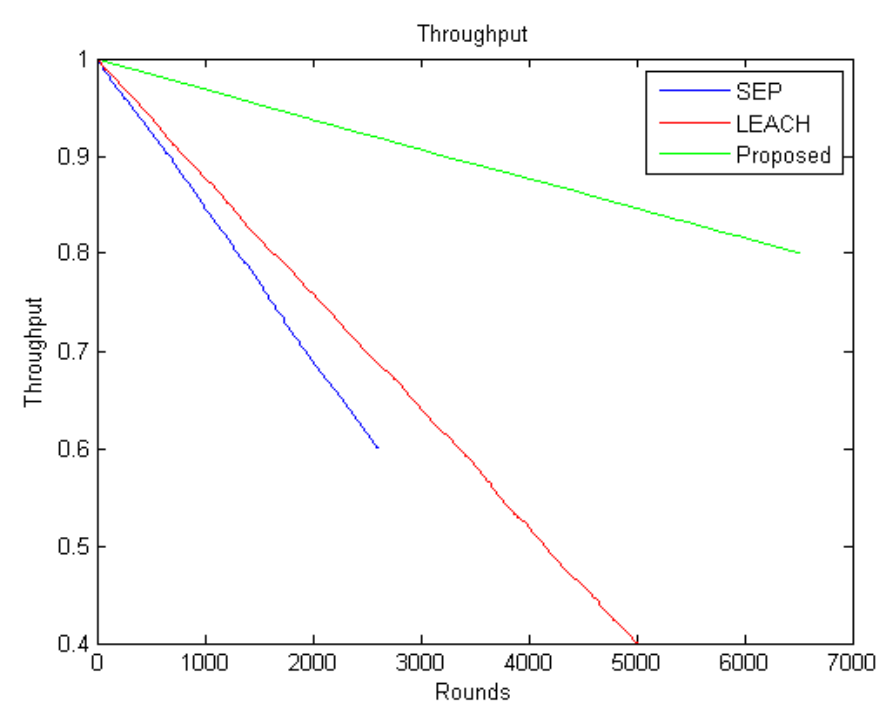

Figure 6 Throughput of different techniques

The figure 6 shows the throughput of different techniques. The graph shows that the throughput of proposed work remains till the completion of rounds that shows the competence. The higher throughput shows the successful message delivery rate over a channel.

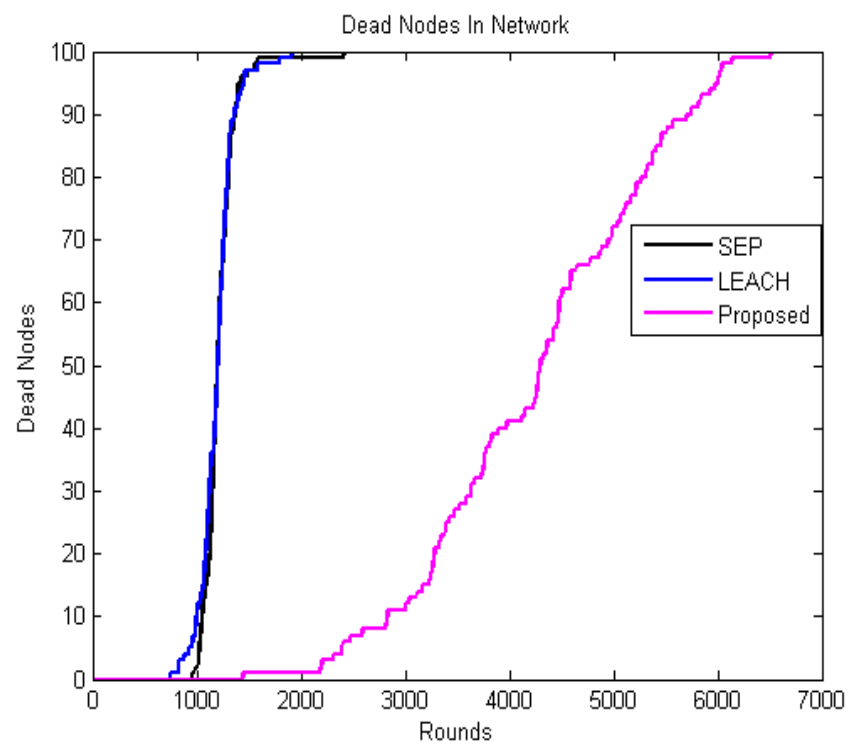

Figure 7 Comparison of dead nodes in the network

The graph 7 shows the efficiency of the proposed work in the terms of dead nodes with respect to different techniques such as SEP, LEACH and proposed. All the nodes are dead in case of SEP and LEACH but in the proposed technique less number of nodes is dead at the end of the transmission.

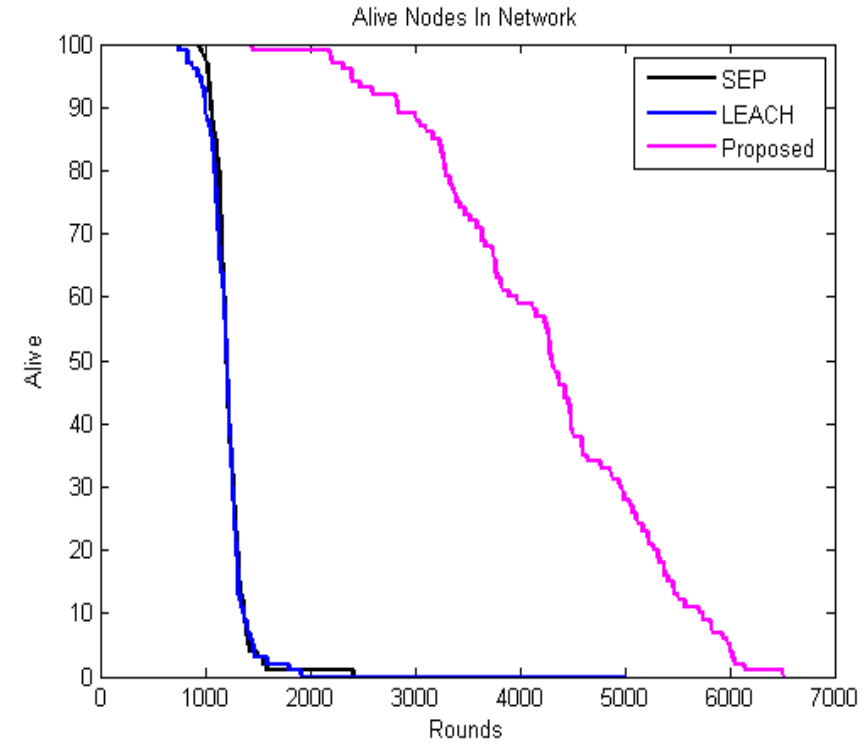

Figure 8 Comparison of Alive nodes in the network versus rounds.

The figure 8 defines the comparison graph of various techniques i.e. SEP, LEACH and proposed work on the basis of number of alive nodes in the network. The graph shows that the proposed system has large number of alive sensor nodes in the WSN as compare to other methods. Number of alive nodes till number of rounds is more stable in case of proposed technique as compared to other techniques.

\section{CONCLUSION AND FUTURE SCOPE}

In this proposed work, existing fuzzy system has enhanced by introducing several parameters such as energy, distance, density, mean value and multi-hoping. These parameters have used to select the clusterhead among the clusters. By introduing these parameters, selection of cluster head becomes accurate. Moreover, the concept of multi-hoping in the proposed work reduces the distance travelled by different nodes to send their data to the sink as nodes forward their data to their nearer nodes. Along with this, as the communication rounds are reducing the energy will be saved for much longer time and can be stable for more communication which is major need of present WSN. Comparison has done using different exiting technqiues to show the efficency of the proposed technique. Simulation results proven the fact that proposed technqiue provides better results in terms of energy , number of alive nodes, dead nodes and throughput in the network. The efficiency and throughput has been increased to $79.69 \%$ and $80 \%$.

The proposed work can be diverisfied into two different fuzzy systems in order to reduce the complexity. In future various techniques can be introduced to enhance the work. 


\section{REFERENCES}

[1] Buyanjargal, “An Energy Efficient Clustering Algorithm for Event-Driven Wireless Sensor Networks (EECED)”, IEEE, pp 1758 - 1763, August, 2009

[2] B. Manzoor£, N. Javaid, "Q-LEACH: A New Routing Protocol for WSNs”, ELSEVIER, Pp 1-6,2013

[3] S Taruna, Megha R. Tiwari,” Event Driven Hierarchical Cluster based Routing Protocol for Wireless Sensor Network”, International Journal of Advanced Research in Computer Science and Software Engineering, Vol. 3, Pp. 549-556,2013

[4] Sharath S.T, Veena N," Quad Clustering Routing Protocol to Enhance the Stability in WSN",IJIRCCE, Vol 2, Pp 39823988,2014

[5] Shounak Chakraborty, “A Noble Approach for Self Leaming and Cluster based Routing Protocol with Power Efficiency in WSN”, IEEE, pp. 773-777, 2014

[6] Yan Sun, "Energy-Efficient Routing Protocol in EventDriven Wireless Sensor Networks”, IEEE, pp 1-5, May 2010

[7] R.Rajeshwari, "Towards Energy Efficient Cluster Based Approach In Wireless Sensor Networks Using Mobile Sink”, IJETCSE, Volume 13 Issue 1, pp 183-186, March 2015

[8] Priya Vyas, Manoj Chouhan," Survey on Clustering Techniques in Wireless Sensor Network", International Journal of Computer Science and Information Technologies, Vol. 5, no. 5, pp. 6614-661, 2014

[9] Meena Malik, Dr. Yudhvir Singh, Anshu Arora, "Analysis of LEACH Protocol in Wireless Sensor Networks", International Journal of Advanced Research in Computer Science and Software Engineering, Volume 3, Pp 178-183, 2013
[10] Lalita Yadav1, Ch. Sunitha, "Low Energy Adaptive Clustering Hierarchy in Wireless Sensor Network (LEACH)”, IJCSIT, Vol 5, Pp 4661-4664

[11] Jianguo SHAN, Lei DONG, Xiaozhong LIAO, Liwei SHAO , Zhigang GAO, Yang GAO, "Research on Improved LEACH Protocol of Wireless Sensor Networks”, Pp 7577,2013

[12] Haitao Zhang and Cuiping Liu, "A Review on Node Deployment of Wireless Sensor Network", I International Journal of Computer Science Issues, Vol. 9, Issue 6, No 3, November 2012

[13] Georgios Smaragdakis Ibrahim Matta Azer Bestavros, "SEP: A Stable Election Protocol for clustered heterogeneous wireless sensor networks", Pp 1-11

[14] Dhanashri V, Ambekar Amol D. Bhoi, R. D Kharadkar, "A Survey on Sensors Lifetime Enhancement Techniques in Wireless Sensor Networks", International Journal of Computer Applications, Vol 107, 2014

[15] Asis Nasipuri, Robert Cox, "Design Considerations for a Large-Scale Wireless Sensor Network for Substation Monitoring”, 5th IEEE International Workshop on Practical Issues in Building Sensor Network Application, Pp 866873,2010

[16] Chu-Fu Wang, “A Network Lifetime Enhancement Method for Sink Relocation and Its Analysis in Wireless Sensor Networks”, IEEE Sensors Journal, Vol. 14, No. 6, pp 19321943, June 2014

[17] Guillermo Molin, Enrique Alba ,El-Ghazali Talbi, “Optimal Sensor Network Layout Using Multi-Objective Metaheuristics”, Journal of Universal Computer Science, vol. 14, no. 15, pp. 2549-2565, 2008

[18] Kemal Akkaya, Mohamed Younis, "A survey on routing protocols for wireless sensor networks”, ELSEVIER, Vol 3, Pp 325-349,2003 